\title{
Early Ultraviolet, Optical, and X\#Ray Observations of the Type IIP SN 2005cs in M51 with Swift
}

\section{Citation}

Brown, Peter J., Luc Dessart, Stephen T. Holland, Stefan Immler, Wayne Landsman, Stephane Blondin, Alexander J. Blustin, et al. 2007. "Early Ultraviolet, Optical, and X\#Ray Observations of the Type IIP SN 2005cs in M51 with Swift." The Astrophysical Journal 659 (2): 1488-95. https:// doi.org/10.1086/511968.

\section{Permanent link}

http://nrs.harvard.edu/urn-3:HUL.InstRepos:41399951

\section{Terms of Use}

This article was downloaded from Harvard University's DASH repository, and is made available under the terms and conditions applicable to Other Posted Material, as set forth at http:// nrs.harvard.edu/urn-3:HUL.InstRepos:dash.current.terms-of-use\#LAA

\section{Share Your Story}

The Harvard community has made this article openly available. Please share how this access benefits you. Submit a story. 


\title{
EARLY ULTRAVIOLET, OPTICAL, AND X-RAY OBSERVATIONS OF THE TYPE IIP SN 2005cs IN M51 WITH SWIFT
}

\author{
Peter J. Brown, ${ }^{1}$ Luc Dessart, ${ }^{2}$ Stephen T. Holland, ${ }^{3,4}$ Stefan Immler, ${ }^{3,4}$ Wayne Landsman, ${ }^{3}$ Stérhane Blondin, ${ }^{5}$ \\ Alexander J. Blustin, ${ }^{6}$ Alice Breeveld, ${ }^{6}$ Gulab C. Dewangan, ${ }^{7}$ Neil Gehrels, ${ }^{8}$ Robert B. Hutchins, ${ }^{5}$ \\ Robert P. Kirshner, ${ }^{5}$ Keith O. Mason, ${ }^{6}$ Paolo A. Mazzali, $,{ }^{9}, 10$ Peter Milne, ${ }^{2}$ \\ Maryam Modjaz, ${ }^{5}$ and Peter W. A. Roming ${ }^{1}$ \\ Received 2006 October 25; accepted 2006 December 16
}

\begin{abstract}
We report early photospheric-phase observations of the Type IIP supernova (SN) 2005 cs obtained by the Swift ultraviolet-optical and X-ray telescopes. Observations started within 2 days of discovery and continued on a regular basis for 3 weeks. During this time the $V$-band magnitude remained essentially constant, while the UV was initially bright, but steadily faded until below the brightness of an underlying UV-bright $\mathrm{H}$ II region. This UV decay is similar to SNe II observed by the International Ultraviolet Explorer. UV grism spectra show the P Cygni absorption of Mg II $2798 \AA$, indicating a photospheric origin of the UV flux. Based on non-LTE model atmosphere calculations with the CMFGEN code, we associate the rapid evolution of the UV flux with the cooling of the ejecta, the peak of the spectral energy distribution (SED) shifting from $\sim 700 \AA \AA$ on June 30 to $\sim 1200 \AA \AA$ on July 5 . Furthermore, the corresponding recombination of the ejecta, e.g., the transition from $\mathrm{Fe}$ III to Fe II, induces a considerable strengthening of metal line-blanketing at and above the photosphere, blocking more and more effectively this fading UV flux. SN 2005cs was not detected in the X-ray band, and the upper limit to the X-ray luminosity yields a limit to the mass-loss rate of the progenitor of $\dot{M} \lesssim 1 \times 10^{-5} \mathrm{yr}^{-1}\left(v_{w} / 10 \mathrm{~km} \mathrm{~s}^{-1}\right)$. Overall, Swift represents a unique opportunity to capture the early and fast evolution of Type II SNe in the UV, providing additional constraints on the reddening, the SED shortward of $4000 \AA$, and the ionization state and temperature of the photon-decoupling regions.
\end{abstract}

Subject headings: galaxies: individual (M51) - ultraviolet: general — X-rays: general

Online material: color figures

\section{INTRODUCTION}

Supernova (SN) $2005 \mathrm{cs}$ was discovered in the Whirlpool galaxy (M51, NGC 5194) by Kloehr et al. (2005) on 2005 June 28.9 (all dates UT). Pastorello et al. (2006) estimate the explosion occurred June 27.5 (JD 2,453,549), based on previous nondetections. Modjaz et al. (2005) classified it from a spectrum as a young SN II on June 30.23, and the observations with the Swift spacecraft (Gehrels et al. 2004) reported here began June 30.9. The photometric and spectroscopic evolution of SN 2005cs is consistent with that of a subluminous SN II-plateau (IIP), with a plateau phase lasting 110 days and low ejecta velocities (Tsvetkov et al. 2006; Pastorello et al. 2006).

A young $\mathrm{SN}$ discovered in a nearby galaxy as well studied as M51 offers a unique opportunity for detailed investigations. The host galaxy has previously been observed at various wavelengths by many different instruments (see, e.g., Calzetti et al. 2005;

\footnotetext{
${ }^{1}$ Pennsylvania State University, Department of Astronomy and Astrophysics, University Park, PA 16802; pbrown@astro.psu.edu.

2 Department of Astronomy and Steward Observatory, University of Arizona, Tucson, AZ 85721.

3 Astrophysics Science Division, X-Ray Astrophysics Branch, NASA Goddard Space Flight Center, Greenbelt, MD 20771.

${ }^{4}$ Universities Space Research Association, Columbia, MD 21044.

${ }^{5}$ Harvard-Smithsonian Center for Astrophysics, Cambridge, MA 01238.

6 Mullard Space Science Laboratory, Department of Space and Climate Physics, University College London, Holmbury St Mary, Dorking, Surrey, RH5 6NT, UK.

${ }^{7}$ Department of Physics, Carnegie Mellon University, Pittsburgh, PA 15213.

8 Astrophysics Science Division, Astroparticle Physics Laboratory, NASA Goddard Space Flight Center, Greenbelt, MD 20771.

9 INAF, Osservatorio Astronomico di Trieste, 34131 Trieste, Italy.

${ }^{10}$ Max-Planck-Institut fur Astrophysik, 85741, Garching, Germany.
}

Dewangan et al. 2005). Examination of preexplosion images from the Hubble Space Telescope (HST) has identified a 7-9 $M_{\odot}$ red supergiant as a plausible progenitor candidate ( $\mathrm{Li}$ et al. 2006; Maund et al. 2005). The position measured using high-resolution images from the Canada-France-Hawaii Telescope and HST is R.A. $=13^{\mathrm{h}} 29^{\mathrm{m}} 52.764^{\mathrm{s}}$ and decl. $=+47^{\circ} 10^{\prime} 36.09^{\prime \prime}(\mathrm{J} 2000.0$; Li et al. 2006).

The quick identification and classification of this SN event allowed for early observations by instruments on the ground and in space while the $\mathrm{SN}$ was still in its early photospheric phase. This is especially critical in the UV, because of the rapid decline in luminosity at short wavelengths as the SN photosphere cools. Because they require space-based observatories, UV data on $\mathrm{SNe}$ are limited, especially at early times. While $I U E$ observed several SNe II, none were of the plateau (IIP) subtype, despite it being the most common type of SNe II. HST observed the SN IIP 1999em at two epochs, starting about 2 weeks after the explosion (Baron et al. 2000). The observations presented in this paper fill an important gap by showing the UV evolution of SN 2005cs during the first few weeks after its explosion.

\section{OBSERVATIONS AND REDUCTIONS}

\subsection{Swift Observations}

Regular observations of SN $2005 \mathrm{cs}$ were made with the Swift spacecraft (Gehrels et al. 2004) between 2005 June 30 and July 23, with a follow-up observation on September 14, utilizing both the X-Ray Telescope (XRT; Burrows et al. 2005) and the Ultraviolet/ Optical Telescope (UVOT; Roming et al. 2005). Images from UVOT and XRT of SN 2005cs and its host galaxy M51 are displayed in Figure 1. The XRT operates in the $0.2-10 \mathrm{keV}$ range 

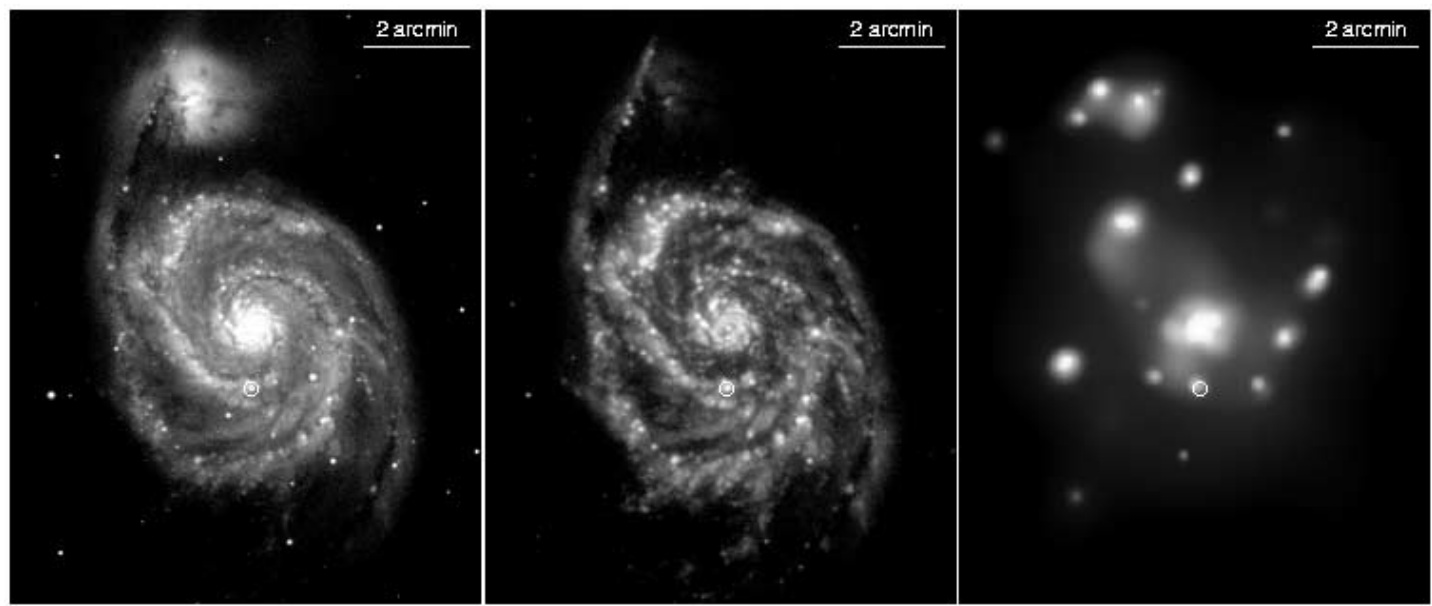

FIG. 1. - Swift UVOT optical (left), UV (middle), and XRT X-ray (right) images of SN 2005cs and its host galaxy, M51. The position of SN $2005 \mathrm{cs}$ is indicated by a white circle of $8^{\prime \prime}$ radius, and the spatial scale, identical for each image, is indicated in the top corner of each panel. The optical image was constructed from summed images from the UVOT $V(1815 \mathrm{~s}$ exposure time), $B(1232 \mathrm{~s})$, and $U(1065 \mathrm{~s})$ filters; the UV image was constructed from the UVOT UVW1 (3038 s), UVM2 (7189 s), and UVW2 $(4703 \mathrm{~s})$ filters and slightly smoothed with a Gaussian filter of 1.5 pixel (FWHM). The $(0.2-10 \mathrm{keV})$ XRT X-ray image was constructed from the merged $33 \mathrm{ks}$ XRT data and adaptively smoothed using the CIAO command csmooth to achieve a signal-to-noise ratio in the range 2.54. [See the electronic edition of the Journal for a color version of this figure.]

with a $18^{\prime \prime}$ half-power diameter at $1.5 \mathrm{keV}$. Pertinent calibration details for UVOT, ${ }^{11}$ including the filter bandpasses and photometric zero points, are given in Table 1.

\subsection{UVOT Photometry}

SN 2005cs is located in a spiral arm of M51, which causes the background to be variable over small spatial scales. There is an underlying $\mathrm{H}$ II region visible in preexplosion GALEX UV images (Bianchi et al. 2005). This means that high-precision photometry will not be possible until SN $2005 \mathrm{cs}$ has faded and the underlying light can be subtracted. It is anticipated that Swift UVOT will reobserve the field of SN $2005 \mathrm{cs}$ in 2007 so that late-time template images of the host galaxy can be obtained. At the early times in which we are most interested here, the SN was much brighter, and the contribution from the $\mathrm{H}$ In region or other contamination is small.

We performed aperture photometry using a circular aperture with a radius of $2^{\prime \prime}(U B V)$ or $4^{\prime \prime}$ (UV filters) centered on the SN. A background sky region was selected by eye, which contained approximately the same structure and light as the region containing the SN. Photometry was done using several background regions selected in this way, and all were found to return similar

\footnotetext{
11 See http://swift.gsfc.nasa.gov/docs/heasarc/caldb/swift/docs/uvot for updated documentation.
}

TABLE 1

Swift UVOT Filter Characteristics

\begin{tabular}{ccccc}
\hline \hline Filter & $\begin{array}{c}\lambda_{c} \\
(\AA)\end{array}$ & $\begin{array}{c}\text { FWHM } \\
(\AA)\end{array}$ & $\begin{array}{c}\text { Zero Point } \\
(\mathrm{mag})\end{array}$ & $\left.\begin{array}{c}\text { Flux Density } \\
\left(10^{-16} \mathrm{ergs} \mathrm{cm}^{-2} \text { counts s}\right.\end{array}\right)$
\end{tabular}

Notes. - The symbol $\lambda_{c}$ refers to the central wavelength of the filter's effective area curve. The flux density is a multiplicative conversion from count rate to flux density, assuming a Vega spectrum. The zero points and flux density factors correspond to a source with a Vega-like spectrum and a count rate of 1 count $\mathrm{s}^{-1}$. photometry. Aperture corrections to the standard UVOT photometry apertures $\left(6^{\prime \prime}\right.$ for $U B V$ and $12^{\prime \prime}$ for UVW1, UVM2, and UVW2) were calculated frame by frame in $U B V$. For the UV filters, a single aperture correction for each filter was computed from a summed image due to the lack of UV-bright, isolated stars in the field of view. The larger source aperture was chosen to minimize errors due to small orbital variations in the point-spread function (PSF).

The count rates were corrected for coincidence loss using the coincidence loss equation in the Swift UVOT Calibration Database (CalDB).$^{12}$ Corrected count rates were transformed to Vega magnitudes using the appropriate photometric zero points in the CalDB. The $U$ and $B$ curves begin near or above the saturation level of UVOT photon-counting detector and will not be considered here. The photometry in the remaining four UVOT filters, three UV filters, and the $V$ band is presented in Table 2 and

12 Available from http://swift.gsfc.nasa.gov/docs/heasarc/caldb/swift.

TABLE 2

Swift UVOT PнотомETRY OF SN 2005cs

\begin{tabular}{ccccc}
\hline \hline JD & UVW2 & UVM2 & UVW1 & $V$ \\
\hline $2,453,552.4 \ldots \ldots$ & $13.46 \pm 0.06$ & $13.01 \pm 0.10$ & $12.89 \pm 0.02$ & $14.55 \pm 0.04$ \\
$2,453,555.1 \ldots \ldots$. & $14.43 \pm 0.06$ & $\ldots$ & $\ldots$ & $14.47 \pm 0.10$ \\
$2,453,557.3 \ldots \ldots$. & $15.02 \pm 0.06$ & $14.97 \pm 0.10$ & $\ldots$ & $14.53 \pm 0.04$ \\
$2,453,557.9 \ldots \ldots$. & $15.22 \pm 0.06$ & $15.20 \pm 0.10$ & $14.52 \pm 0.03$ & $14.55 \pm 0.06$ \\
$2,453,558.8 \ldots \ldots$. & $15.75 \pm 0.07$ & $15.62 \pm 0.11$ & $14.80 \pm 0.03$ & $14.65 \pm 0.04$ \\
$2,453,560.0 \ldots \ldots$. & $16.02 \pm 0.07$ & $16.04 \pm 0.11$ & $15.23 \pm 0.03$ & $14.57 \pm 0.04$ \\
$2,453,560.6 \ldots \ldots$. & $16.26 \pm 0.07$ & $16.14 \pm 0.11$ & $15.43 \pm 0.04$ & $14.58 \pm 0.06$ \\
$2,453,562.0 \ldots \ldots$. & $16.33 \pm 0.07$ & $16.26 \pm 0.11$ & $15.72 \pm 0.04$ & $14.55 \pm 0.06$ \\
$2,453,562.8 \ldots \ldots$. & $16.62 \pm 0.08$ & $16.22 \pm 0.11$ & $15.78 \pm 0.05$ & $14.63 \pm 0.06$ \\
$2,453,565.0 \ldots \ldots$. & $16.46 \pm 0.08$ & $16.36 \pm 0.12$ & $15.94 \pm 0.06$ & $14.55 \pm 0.08$ \\
$2,453,567.0 \ldots \ldots$. & $16.50 \pm 0.07$ & $16.24 \pm 0.11$ & $15.94 \pm 0.04$ & $14.65 \pm 0.05$ \\
$2,453,571.0 \ldots \ldots$. & $16.62 \pm 0.08$ & $16.49 \pm 0.12$ & $16.11 \pm 0.05$ & $14.73 \pm 0.08$ \\
$2,453,573.5 \ldots \ldots$. & $16.50 \pm 0.08$ & $16.32 \pm 0.12$ & $16.23 \pm 0.06$ & $14.76 \pm 0.09$ \\
$2,453,628.5 \ldots \ldots$. & $16.67 \pm 0.07$ & $16.39 \pm 0.11$ & $16.44 \pm 0.04$ & $14.86 \pm 0.03$
\end{tabular}

Notes.-The observation time is given as the Julian Date (JD) of the middle of the set of exposures. These values have not been corrected for extinction. The errors given are $1 \sigma$ statistical errors. 


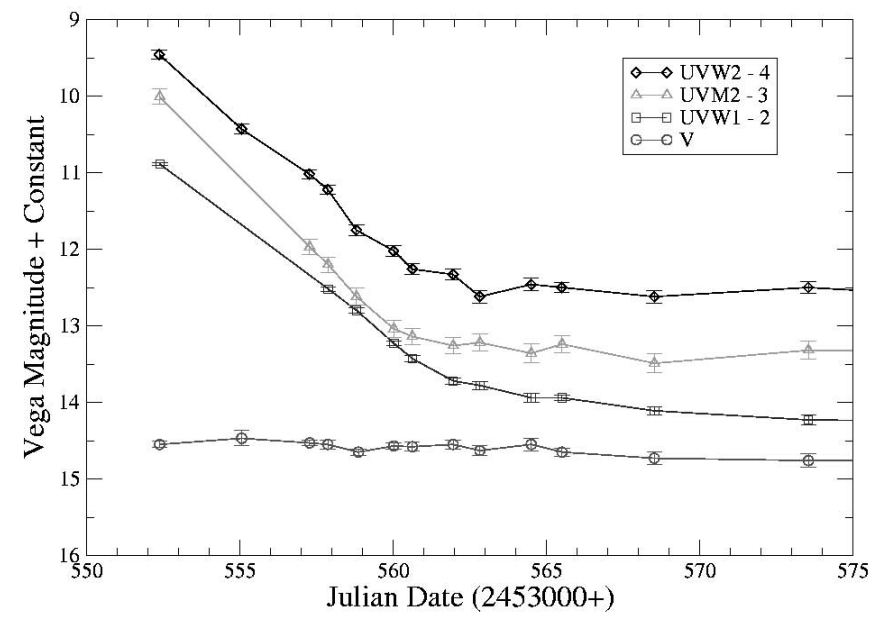

FIG. 2.- - Light curves of SN 2005 cs obtained by UVOT in the three UV filters and the $V$ band. For clarity, the UV curves have been shifted by a constant offset given in the legend. The steep decay in the UV levels off as the SN fades below the brightness of the underlying $\mathrm{H}$ in region. [See the electronic edition of the Journal for a color version of this figure.]

displayed in Figure 2. These values have not been corrected for extinction. The errors (given in Table 2 or displayed in Fig. 2) are the $1 \sigma$ statistical errors only and do not include the systematic errors in the photometric zero-point calibration (given in Table 1).

\subsection{UVOT Grism Data}

Swift UVOT also observed SN 2005cs several times using its spectroscopic grisms. These observations are listed in Table 3. The wavelength scale could be shifted by up to $30 \AA$ due to the difficulty of fixing the scale on the saturated zeroth-order spectrum, especially at early times when the UV flux was greatest. There is also a large uncertainty in the background subtraction due to the bright underlying galaxy.

The extracted spectra were smoothed using a running average of 10 points $(\sim 20 \AA)$ and scaled to match contemporaneous UVOT photometry (using the flux density conversion factors of Table 1). The resulting spectra from four epochs are displayed in Figure 3. The first epoch spectrum is a composite of the UV grism and $V$ grism spectra, spliced near $2900 \AA$, in order to avoid order overlap in the UV grism. The UV grism observation from 6 July contains the zeroth order of another field star near $2450 \AA$, and the affected region has been removed from the spectrum.

\subsection{XRT Data}

All XRT data, from June 30 to September 14, were merged into a single $33.0 \mathrm{ks}$ observation to search for X-ray emission from

TABLE 3

Swift UVOT Grism Observations

\begin{tabular}{|c|c|c|c|}
\hline Observation Sequence & $\begin{array}{l}\text { Date } \\
\text { (UT) }\end{array}$ & $\begin{array}{c}\text { Exposure } \\
\text { (s) }\end{array}$ & Grism \\
\hline $00030083007 \ldots$ & 2005 Jul 3 & 2078.1 & $V$ \\
\hline 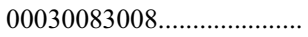 & 2005 Jul 3 & 2078.5 & UV \\
\hline $00030083012 \ldots \ldots \ldots \ldots \ldots \ldots$ & 2005 Jul 6 & 2137.9 & UV \\
\hline $00030083017 \ldots \ldots$ & 2005 Jul 8 & 2017.7 & UV \\
\hline $00030083023 \ldots \ldots \ldots \ldots \ldots \ldots$ & 2005 Jul 11 & 2077.7 & UV \\
\hline $00030083027 \ldots \ldots \ldots \ldots \ldots \ldots$ & 2005 Jul 13 & 1788.3 & UV \\
\hline $00030083035 \ldots \ldots \ldots \ldots \ldots \ldots$ & 2005 Jul 19 & 1955.2 & UV \\
\hline
\end{tabular}

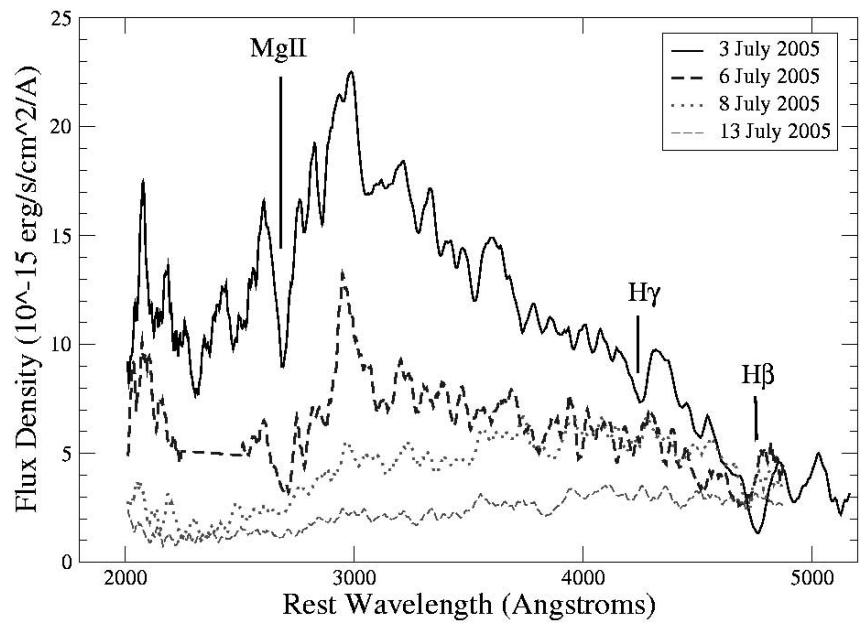

FIG. 3.- Grism spectra of SN 2005cs obtained by UVOT, smoothed and scaled to contemporaneous UVOT photometry. The first spectrum is a composite of UV and $V$ grism spectra (spliced at $2900 \AA$ ), and the others are from the UV grism. We highlight a few features, which we associate with the absorption part of $\mathrm{P}$ Cygni profiles for $\mathrm{Mg}$ II $2798 \AA, \mathrm{H} \beta$, and $\mathrm{H} \gamma$; these Doppler-broadened absorptions are consistent with other optical features, whose modeling supports a formation within the fast-expanding SN ejecta, rather than in the CSM (see $\S 3.3$ for discussion). [See the electronic edition of the Journal for a color version of this figure.]

SN 2005cs. However, the bright [integrated flux between 0.2 and $\left.10 \mathrm{keV}, f_{0.2-10}=(3.3 \pm 0.7) \times 10^{-14} \mathrm{ergs} \mathrm{cm}^{-2} \mathrm{~s}^{-1}\right]$ and nearby $\left(8^{\prime \prime}\right.$ offset) X-ray flash source detected in sequence number 00030083011 (Immler et al. 2005a) severely affects an estimate of the amount of emission from SN $2005 \mathrm{cs}$, because this source is within the XRT source extraction aperture $\left(18^{\prime \prime}\right.$ half-power diameter at $1.5 \mathrm{keV})$. We therefore excluded all observations obtained on the day the X-ray flash was observed (2005 July 6 , sequence numbers $00030083010 / 11 / 12 / 13$ ) to minimize contamination, leaving $26.8 \mathrm{ks}$ of exposure time in the final merged image used below.

The $26.8 \mathrm{ks}$ image shows no X-ray source at the position of the X-ray flash and no source at the position of SN 2005cs. We therefore extracted on-source counts from a circular extraction region with a radius of 10 pixel $\left(23.6^{\prime \prime}\right)$ and subtracted the background from an annulus centered on the position of SN $2005 \mathrm{cs}$ to account for diffuse emission from the galaxy. A $3 \sigma$ upper limit to the $0.2-10 \mathrm{keV}$ count rate of $1.7 \times 10^{-3}$ counts $\mathrm{s}^{-1}$ is obtained, which corresponds to an unabsorbed X-ray flux of $f_{0.2-10} \lesssim$ $9.0 \times 10^{-14} \mathrm{ergs} \mathrm{cm}^{-2} \mathrm{~s}^{-1}$.

\subsection{XMM-Newton EPIC Data}

SN 2005cs was observed with XMM-Newton on 2005 July 1 (ObsID 0212480801), as a Target of Opportunity (PI: S. Immler) to search for prompt X-ray emission from the SN. Data processing and analysis were performed with SAS $6.5 .0^{13}$ and the latest calibration constituents. After screening of the EPIC pn and MOS data for periods with a high background, cleaned exposure times of $28.1 \mathrm{ks}$ for the pn and $33.8 \mathrm{ks}$ for each of the two MOS instruments were obtained.

Inspection of the EPIC images showed that the X-ray "flash" serendipitously observed by Swift XRT during some of the observations (Immler et al. 2005a) is also present in the data obtained quasi-simultaneously with $X M M-N e w t o n$ EPIC. Since the offset of this bright X-ray source from the position of SN $2005 \mathrm{cs}$ $\left(8^{\prime \prime}\right)$ is smaller than the PSF of the XRT+EPIC $\left(15^{\prime \prime}\right.$ half-energy

13 See http://xmm.vilspa.esa.es/external/xmm_sw_cal/sas.shtml. 
width), no reliable upper limits to the X-ray flux of SN 2005cs can be established from the $X M M$-Newton X-ray data. The $X M M$ Newton EPIC data will therefore not be further used, and we will rely on the Swift XRT data to study the X-ray emission from SN $2005 \mathrm{cs}$.

\section{DISCUSSION}

\subsection{Ultraviolet Light Curves}

SN 2005cs was initially extremely bright in the UV, outshining the nucleus of M51 by over 2 mag. The blue UV-optical colors of SN 2005cs were used to photometrically type SNe 2006at (Brown \& Immler 2006) and 2006bc (Immler \& Brown 2006) as young SNe II. The UV faded quickly until it fell below the brightness of an underlying $\mathrm{H}$ II region about 10 days after the explosion. While the decay slope generally steepens at shorter wavelengths (see Li et al. [2006], Tsvetkov et al. [2006], or Pastorello et al. [2006] for the optical curves), the decline in UVM2 is actually steeper $\left(0.38 \mathrm{mag} \mathrm{day}^{-1}\right)$ than the filters on either side, UVW1 $\left(0.31 \mathrm{mag} \mathrm{day}^{-1}\right)$ and UVW2 $\left(0.34 \mathrm{mag} \mathrm{day}^{-1}\right)$. This is likely caused by the strong $\mathrm{Fe}$ III and the strengthening $\mathrm{Fe}$ II lines concentrated within the UVM2 bandpass.

For a comparison with other SNe II in a nearby UV wavelength regime, we created a new UVW1 light curve for SN 2005cs by assuming that the magnitude in the last epoch $(\sim 80$ days after explosion) corresponds to the underlying background and subtracting that flux from the early UVW1 data. This might overestimate the background caused by a residual contribution from the $\mathrm{SN}$, but this is not expected to be significant, because of the continued fading seen in more recent UV observations of SNe II, such as SN 2006bp (Immler et al. 2006). This new UVW1 curve is compared to other UV observations of SNe II in Figure 4.

Several SNe II were observed by IUE, including several with multiple epochs (more than four observations: SNe 1979C, 1980K, $1987 \mathrm{~A}$, and 1993J). For comparison, we use the $m_{275}$ magnitudes given by Cappellaro et al. (1995), which are calculated by convolving the HST F275W filter (central wavelength $2770 \AA$ and width $594 \AA$ \&; Nota et al. 1996) with the IUE spectra and calibrated so Vega would have $m_{275}=0$. Due to the quantity of data, only an early selection from SN 1987A was used. Any magnitudes from spectra marked as saturated were also left out, as were the later spectra of SN 1993J, which were contaminated by sunlight. We left the IUE magnitudes unshifted, as the difference in apparent magnitude between the brighter SNe observed by IUE and SN $2005 \mathrm{cs}$ is enough to separate their respective curves.

To make sure the comparison of the UV light-curve shapes with the UVW1 and $m_{275}$ filters is valid, we performed spectrophotometry on the IUE spectra of SN 1987A (Pun et al. 1995) using the IRAF SBANDS command. A selection of spectra from the Long Wave Prime (LWP) camera (1975-3300 Ä; Pun et al. 1995 ) were convolved with the UVW1 effective area curves to simulate how SN 1987A would have appeared in the UVW1 filter. The light curves of the UVW1 spectrophotometry and $m_{275}$ were very similar.

While the fading behavior in the UV is common among these $\mathrm{SNe}$, it is interesting to note how varied the early optical ( $V$ band) behavior is for these SNe during the same time period. SN 1979C (Panagia et al. 1980; Barbon et al. 1982) and SN 1980K (Buta 1982), both II-linear SNe (IIL), were discovered after maximum light and were thus fading in the optical. SN 1993J (Richmond et al. 1994), a Type IIb, was caught soon after explosion and showed the brightness declining, reaching a local minimum about 8 days before rising again as the photosphere receded to layers influenced by nonthermal excitation due to the radioactive

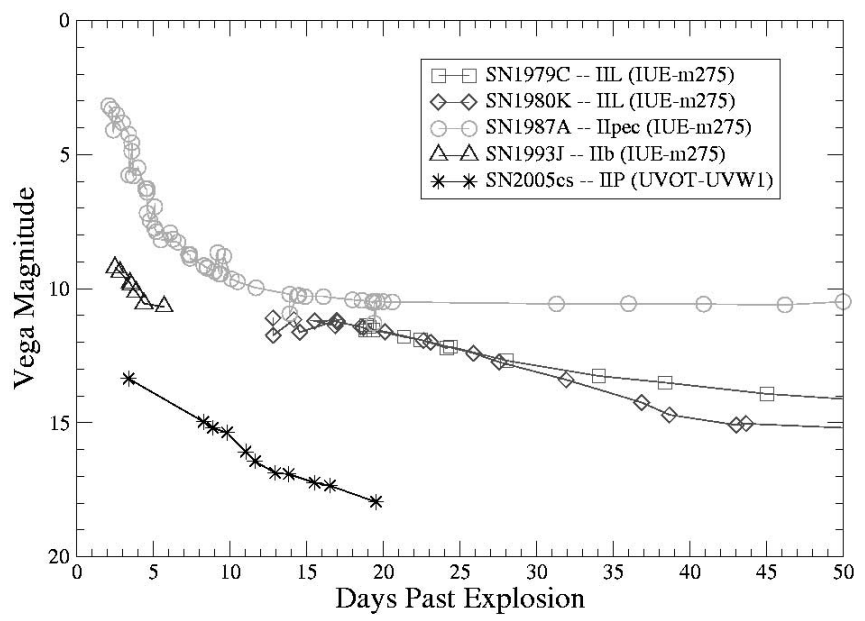

FIG. 4.-Comparison of SNe Type II observed by IUE and Swift UVOT. An early UV decay is seen in all these SNe, despite the differences in subclass or optical behavior. [See the electronic edition of the Journal for a color version of this figure.]

decay of unstable isotopes of nickel and cobalt. SN 1987A (Pun et al. 1995), a peculiar Type II, was mostly rising during this period. As shown in Figure 2, SN 2005cs had a nearly constant $V$ magnitude during this period, the distinguishing characteristic of a SN II-plateau. In all cases, the UV and optical light curves must have begun with a rapid rise at the time of shock breakout, which is not usually observed. But while the optical curves vary enough to separate SNe II into subclasses based on their lightcurve shape (Barbon et al. 1979), differences arising from subclass or emission mechanism are not as clear in the UV.

\subsection{UV Spectra}

The first two UV spectra displayed in Figure 3 show strong features also seen in the HST spectra of SN 1999em (Baron et al. 2000). Even after smoothing, the spectra still contain significant noise, as evidenced by features in the optical regions, which are not apparent in contemporaneous ground-based optical spectra (Pastorello et al. 2006). Because of this and the $\sim 30 \AA$ uncertainty in the wavelength scale, we do not want to overinterpret line identifications or velocities. We can identify a few strong lines, namely, $\mathrm{H} \beta, \mathrm{H} \gamma$, and the $\mathrm{Mg}$ II 2798 multiplet, whose strong and broad absorption features are marked. Overlapping absorption lines, particularly of $\mathrm{Fe}$ III, $\mathrm{Fe}$ II, and $\mathrm{Mg}$ II, remove much of the UV light (see, e.g., Lucy 1987; Branch 1987; Mazzali \& Lucy 1993; Dessart \& Hillier 2005, 2006). A complete census of the various ions and the spectral regions where they affect the emergent light is computed for two epochs bracketing the first grism observation (see discussion and Fig. 5), generated by performing a formal solution to a converged CMFGEN model, but accounting only for the bound-bound transitions of a single ion. This neglects potential nonlinear effects and line overlap, but offers an instructive illustration of the cumulative effects of line blanketing by disentangling all contributing species. Note how little line emission appears above the UV continuum and that the resulting "emission" features in the UV coincide with spectral regions of reduced line blanketing. The later UV spectra are of lesser quality, but show the UV continuum becoming increasingly fainter.

The broad absorption around $2700 \AA$ is consistent with the formation of $\mathrm{Mg}_{\text {II }} 2798 \AA$ in the $\mathrm{SN}$ ejecta, i.e., at and above the SN photosphere at that time, and we associate it with the absorption part of the corresponding P Cygni profile of this strong line (see $\S 3.3$ ). This suggests that the UV emission from SN 2005cs 

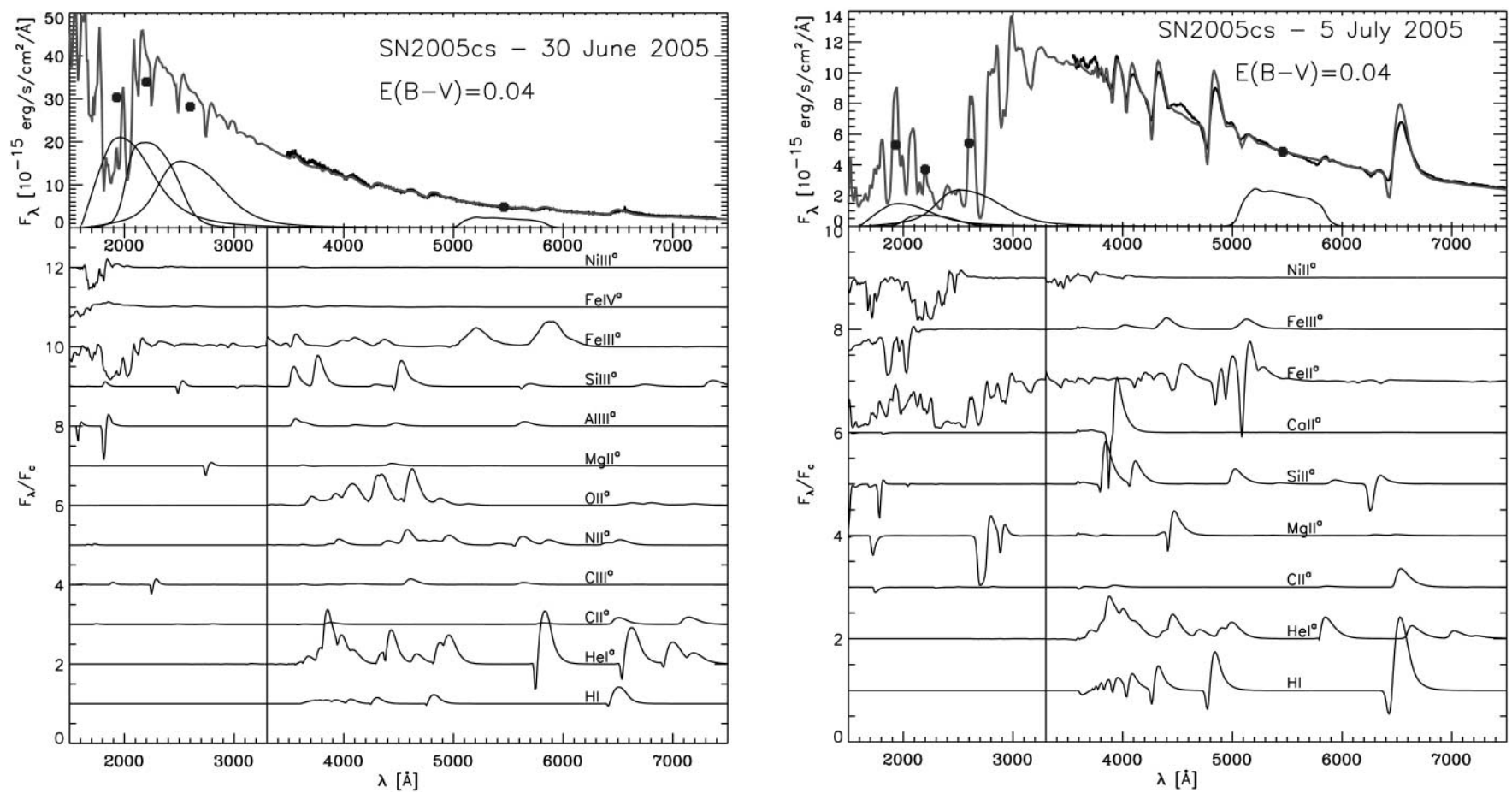

FIG. 5.-Left: Comparison between the photometric UVOT (crosses), spectroscopic optical observations from CfA for SN $2005 \mathrm{cs}$ on the 2006 June 30 , and a reddened $[E(B-V)=0.04]$ synthetic spectrum computed with CMFGEN (line) using the procedure followed for the Type IIP SN 1999em and described in Dessart \& Hillier (2006). Model parameters are $L_{*}=2.7 \times 10^{8} L_{\odot}, T_{\text {phot }}=15,750 \mathrm{~K}, R_{\text {phot }}=2 \times 10^{14} \mathrm{~cm}, v_{\text {phot }}=6900 \mathrm{~km} \mathrm{~s}^{-1}, \rho_{\text {phot }}=2.5 \times 10^{-13} \mathrm{~g} \mathrm{~cm}^{-3}$, and $n=20$. The synthetic flux, $20 \%$ lower than observed for an adopted distance of $8 \mathrm{Mpc}$, is renormalized at $6000 \AA$. UVOT fluxes are scaled so that the $V$-band flux matches the observed flux at $5460 \AA$. For both figures, we include in the lower panel the synthetic spectra obtained by including bound-bound transitions only of the selected ions (and normalized by the continuum), thereby illustrating the sources line blanketing, in particular in the UV range. Only the ions leaving a nontrivial mark are included. Moreover, for ions marked with a superscript "a," we apply a magnification of 10 beyond $3300 \AA$ to enhance the visibility of the weak features in the optical range. Right: Same as left for the observations of SN $2005 \mathrm{cs}$ on the 2005 July 5 and a reddened synthetic CMFGEN spectrum, whose corresponding model parameters are $L_{*}=1.5 \times 10^{8} L_{\odot}, T_{\text {phot }}=8200 \mathrm{~K}$, $R_{\text {phot }}=4.2 \times 10^{14} \mathrm{~cm}, v_{\text {phot }}=5200 \mathrm{~km} \mathrm{~s}^{-1}, \rho_{\text {phot }}=6.4 \times 10^{-14} \mathrm{~g} \mathrm{~cm}^{-3}$, and $n=10$. The synthetic flux, $10 \%$ lower than observed for an adopted distance of $8 \mathrm{Mpc}$, is renormalized at $6000 \AA$, and the UVOT fluxes scaled to match the $V$ band at $5460 \AA$. We also overplot the UVW2, UVM2, UVW1, and $V$ filter bandpasses, scaled in proportion to the observed flux and to avoid overlap with the spectra. For both epochs, the (weak) UV brightness of the underlying $\mathrm{H}$ II at $\sim 80$ days has been subtracted. (See text for discussion.) [See the electronic edition of the Journal for a color version of this figure.]

originates from the photospheric layers of the fast-expanding ejecta, rather than from interaction with the circumstellar medium (CSM). SNe 1979C, 1980K, and 1993J all showed blueshifted emission from Mg II and had smoother UV spectra (see, e.g., Panagia et al. 1980; Fransson 1984; Immler et al. 2005b; Jeffery et al. 1994, 2006). SN 1987A, on the other hand, exhibited a $\mathrm{P}$ Cygni profile for $\mathrm{Mg}$ II and no evidence for strong CSM interaction at early times (Kirshner et al. 1987; Pun et al. 1995). The addition of our spectra in Figure 3 here to Figure 3 of Jeffery et al. (1994) gives a spectroscopic juxtaposition of the same $\mathrm{SNe}$ compared photometrically in our Figure 4.

\subsection{Comparison with Non-LTE Model Atmospheres}

We report on a preliminary quantitative analysis of the UVOT photometric evolution and optical ground-based spectroscopy covering the first 2 weeks after discovery. Detailed results of this study will be presented in a forthcoming paper (L. Dessart et al. 2007 , in preparation).

We employ the non-LTE model atmosphere code CMFGEN (Hillier \& Miller 1998; Dessart \& Hillier 2005) and follow the same approach as Dessart \& Hillier (2006) for their analysis of SN $1999 \mathrm{em}$. The version of the code used assumes a spherically symmetric (one-dimensional), steady state, chemically homogeneous, homologously expanding ejecta with a density power law of exponent $n$. A key feature of our approach is that we set the inner boundary luminosity of the model (at a Rosseland optical depth of $\sim 50-100$ ) so that the emergent synthetic flux matches the observed flux. To do this, we require the distance to SN $2005 \mathrm{cs}$, which we take as $8 \mathrm{Mpc}$, the rough average of various distance estimates to the host galaxy M51 or sources within it. These distance estimates used H II regions (9.6 Mpc; Sandage \& Tammann 1974), young stellar associations (6.91 Mpc; Georgiev et al. 1990), planetary nebulae (8.4 Mpc, revised to 7.6 Mpc; Feldmeier et al. 1997; Ciardullo et al. 2002), surface brightness fluctuations (7.7 Mpc; Tonry et al. 2001), the spectral-fitting expanding atmosphere method (6.0 Mpc; Baron et al. 1996), and more recently the expanding atmosphere method and standard candle method using SN 2005cs itself (7.3 Mpc; Takáts \& Vinkó 2006). We redden the synthetic SED computed by CMFGEN using the law of Cardelli et al. (1988) and $E(B-V)=0.04$, which offers the best fit to the observations, and renormalize them to the observed flux at $6000 \AA$ (the flux offset between synthetic and observed spectra is small and $\sim 10 \%-20 \%$ ).

We assume an (homogeneous) ejecta composition that differs somewhat from that Dessart \& Hillier (2006) used for SN 1999em. Indeed, $\mathrm{O}_{\text {II }}$ lines present in the June 30 spectrum at $\sim 4600 \AA$ required a higher oxygen abundance (Baron 2007). We obtain satisfactory fits at all times using $\mathrm{H} / \mathrm{He}=5, \mathrm{C} / \mathrm{He}=0.0004$, $\mathrm{N} / \mathrm{He}=0.0013$, and $\mathrm{O} / \mathrm{He}=0.0016$, in agreement with the recent determination of the average surface composition of Galactic B supergiants (Crowther et al. 2006), together with a solar metallicity for the other elements.

To document the early color evolution of the SED of SN 2005cs, we focus on only two dates, June 30 and July 5, complementing 

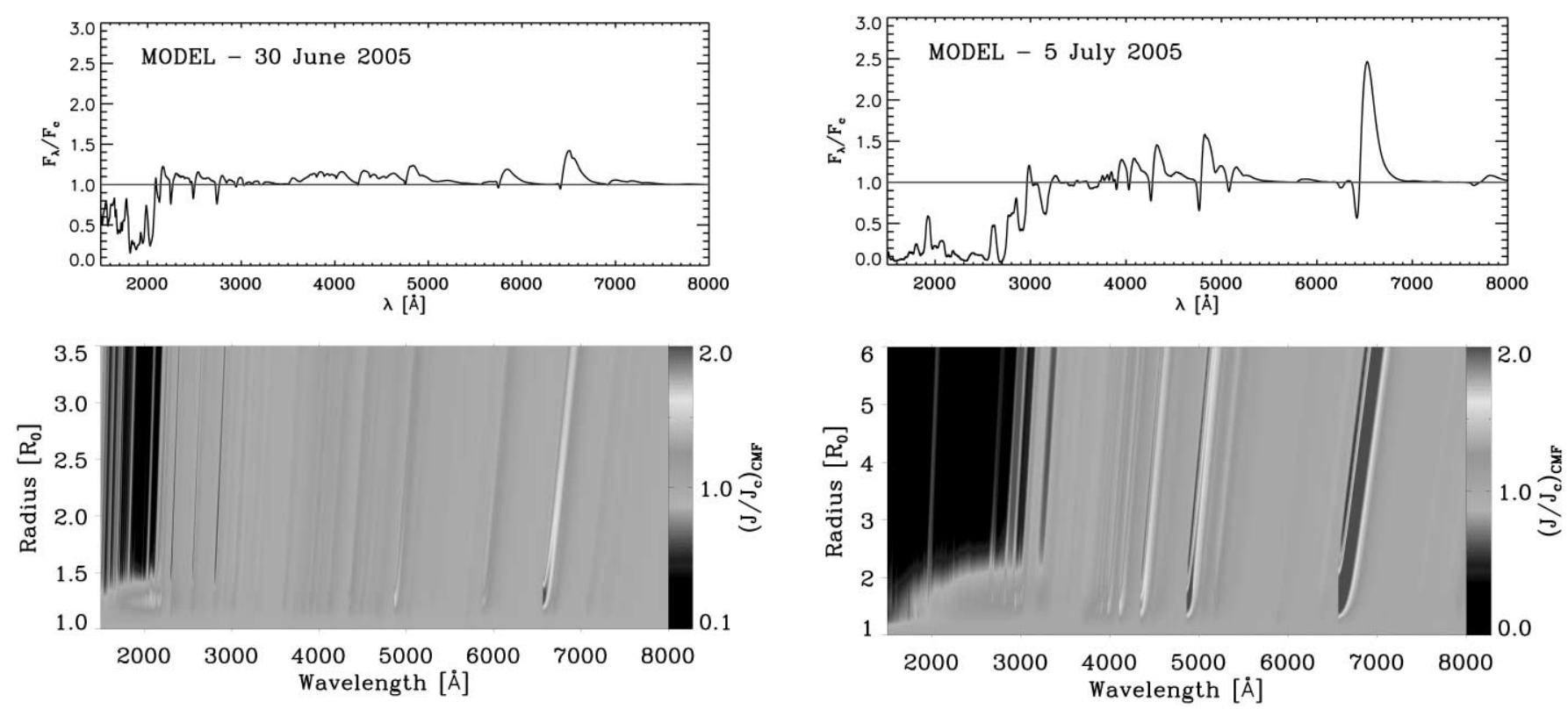

FIG. 6.-Left: Radial variation (in the comoving frame) of the mean intensity normalized to the continuum mean intensity over the UV and optical ranges (bottom panels) for the June 30 CMFGEN model. Top: Synthetic emergent spectrum, normalized to the continuum spectrum. Right: Same as left, but this time for the July 5 CMFGEN model. [See the electronic edition of the Journal for a color version of this figure.]

UVOT observations with optical spectra obtained by the Center for Astrophysics (CfA) SN Group (for June 30) and Pastorello et al. (2006; for July 5) - the full time sequence will be shown in the follow-up analysis to this paper. We show in Figure 5 a comparison for these two dates of the observed UVOT (crosses) and optical spectra (black curve) of SN 2005cs with reddened synthetic spectra computed with CMFGEN. At the bottom of each plot is a ladder plot of spectra (normalized by the continuum) showing the contribution of bound-bound transitions of individual ions. To fit the observations, we primarily vary the base radius and luminosity to modulate the ionization state of the ejecta. This changes, in a nonlinear way, the sources of opacity, thereby modifying the line strengths. It also changes the hardness of the SED at the thermalization layer (which lies above the inner boundary of the model), where it resembles that of a blackbody. Hence, this modifies the slope of the continuum as well. We also change the density exponent $n$, adopting a very high value of 20 for the June 30 model. This represents an extremely steep density decrease with radius, but is necessary in order to reproduce the very weak optical features. A steep density profile was also required to fit early observations of SN 1993J (Baron et al. 1995). A lower value of 10 is used to reproduce the observations of July 5 . We obtain satisfactory fits for a reddening of 0.04 , which we adopt for all dates. Discordant flux distributions between quasi-simultaneous observations of Pastorello et al. (2006) and the CfA suggest that the flux is not very accurate below $4000 \AA$, likely due to the CfA spectra not being taken at the parallactic angle. This is a region where spectra would best constrain the reddening, ionization, and temperature of the ejecta. The corresponding uncertainty in the slope suggests that the reddening could be as low as 0 , but could not be higher than 0.1 without setting stringent requirements on the spectral modeling. Either way, the reddening is low, with a value equal or lower than that adopted by Pastorello et al. (2006), namely, $E(B-V)=0.11$. Baron (2007) also found the reddening to be small and adopted the Galactic value for the extinction from the Schlegel dust maps $[E(B-V)=0.035$; Schlegel et al. 1998].
We find that the density distribution flattens considerably in the continuum and line formation region over that week, described here as a change in the density exponent from $n=20$ to 10 . This reproduces the changing optical line features, which appear systematically weak in the first spectrum, but considerably stronger in the second. Given the numerous constraints on our modeling, varying only the density exponent can lead to the desired reduction of line fluxes in the first epoch. The corresponding flattening of the density profile over the course of 1 week does not constitute a puzzle. As demonstrated by Dessart \& Hillier (2005), line formation in Type II SN is localized to a narrow spatial region above the photosphere, and thus, no sizeable flux emission occurs beyond a few tens of percent beyond the photospheric velocity. On 2005 July 5, $\sim 90 \%$ of the $\mathrm{H} \alpha$ flux (the strongest optical line) received from SN $2005 \mathrm{cs}$ falls within a range of lineof-sight velocities of $\pm 5000 \mathrm{~km} \mathrm{~s}^{-1}$, thus well below the photospheric velocity on June 30, for which we adopt the steeper density slope. Another point (S. Woosley 2006, private communication) is that the ejecta swept up by the shock remains in a dynamical phase up to 1 week after breakout. Homologous expansion is not reached until after about 1 week. This suggests that interpreting and comparing ejecta velocities inferred spectroscopically during the first week of explosion is not straightforward.

Besides a flattening of the density distribution, we also find that the ionization and temperature change significantly between these two dates, following cooling through expansion of the ejecta, as well as radiation from the surface layers. We infer a drop of the photospheric temperature from $15,750 \mathrm{~K}$ down to $8200 \mathrm{~K}$ in that period. (We define the photosphere as the ejecta location where the inward integrated optical depth at $5000 \AA$, including bound-free, free-free, and electron-scattering opacity processes, is equal to two-thirds.) The corresponding constraints from the observations are the fast-changing UV flux and the strengthening of line blanketing in the UV and, more modestly, in the optical. Line identifications apply here in the same way as for SN 1999em (Dessart \& Hillier 2006), but with a few alterations. The first spectrum is likely closer to the explosion date (see Dessart \& Hillier 
2006), showing much weaker lines and a steeper spectrum than for SN 1999em. The ejecta of SN 2005cs is also slower, so that we confirm not only the presence of $\mathrm{N}$ II lines (Dessart \& Hillier 2006), but also that of O II at $4600 \AA$. The Si II $6355 \AA$ line is also well resolved, while it overlapped with the $\mathrm{H} \alpha$ trough in SN 1999em (Dessart \& Hillier 2006; Leonard et al. 2002b) or SN 1999gi (Leonard et al. 2002a).

The temporal evolution of the flux distribution reflects first the cooling of the photosphere. At the base of our two CMFGEN models (corresponding to a Rosseland optical depth of 70 and 73 , or a radius of 1.6 and $4.176 \times 10^{14} \mathrm{~cm}$ for the two selected dates), the electron temperature varies from $40,750 \mathrm{~K}$ down to $23,670 \mathrm{~K}$, and the corresponding flux distributions, which match blackbodies at such depths, peak at $710 \AA$ and $1220 \AA$. We thus see that the intrinsic photon distribution softens significantly, although it still peaks in the UV. The flux distribution that emerges peaks further to the red due to the intervening blocking of light in the UV between the thermalization layer, where the photon distribution is characterized, and layers where it escapes freely. We illustrate this effect in Figure 6 by showing the radial variation in the comoving frame (the radiation field appears redshifted with increasing radius) of the mean intensity $\left(F_{\lambda}\right)$ normalized to the continuum mean intensity $\left(F_{c}\right.$; bottom panels $)$ for the June 30 (left) and the July 5 (right) models. Here the continuum mean intensity is computed by solving the radiative transfer equation for the processes associated with the continuum only. At the base, $F_{\lambda} / F_{c}$ is unity, but as we cross the photosphere (at $\sim 1.4 R_{0}$ for June 30 and $\sim 2 R_{0}$ for July 5 , where $R_{0}$ is the base model radius), this ratio suddenly drops below unity in the UV, while showing the appearance of many lines in the optical. At such early times, optical lines are mostly of $\mathrm{H}$ I and $\mathrm{He} \mathrm{I}$, with weaker contributions from $\mathrm{N}$ II and $\mathrm{O}$ II. In the top panel, we show the emergent synthetic spectrum (normalized to the continuum) for comparison. Hence, we see that line blanketing plays a strong role in the UV, but it operates on a photon distribution that becomes more and more depleted in the UV as time progresses. Our model atmosphere calculations support a purely photospheric origin for the observed flux, both for the UV and the optical ranges, and thus do not suggest any sizeable CSM contribution (ignoring the underlying $\mathrm{H}$ II region contribution). These two combined effects, the reddening of the continuum and the strengthening of the line blanketing, explain the color evolution of SN $2005 \mathrm{cs}$ and in particular confirm the "thermal" photospheric origin of this radiation. Work is underway to interpret quantitatively the absolute flux levels observed, with links to the explosion energy and the ejecta kinematics.

\subsection{X-Ray Results}

The upper limit from the merged $26.8 \mathrm{ks}$ XRT observation (after screening out the day of the X-ray flash) corresponds to an unabsorbed X-ray flux and luminosity of $f_{0.2-10} \lesssim 9.0 \times$ $10^{-14} \mathrm{ergs} \mathrm{cm}^{-2} \mathrm{~s}^{-1}$ and $L_{0.2-10} \lesssim 8.4 \times 10^{38} \mathrm{ergs} \mathrm{s}^{-1}$, respectively, for an assumed thermal plasma spectrum with a temperature of $10 \mathrm{keV}$, an absorbing foreground column density of $1.6 \times 10^{20} \mathrm{~cm}^{-2}$ (Dickey \& Lockman 1990) and a distance of
$8 \mathrm{Mpc}$. SNe IIP have been detected in X-rays in the past (SN 1999em, Pooley et al. 2002; SN 1999gi, Schlegel 2001; SN 2004dj, Pooley \& Lewin 2004), but usually at lower luminosities (typically around $10^{38} \mathrm{ergs} \mathrm{s}^{-1}$ ) than our upper limits for SN 2005cs. But these observations do rule out an X-ray-bright SN more luminous than SN 1994W $\left(8 \times 10^{38} \mathrm{ergs} \mathrm{s}^{-1}\right)$, a Type IIP with optical evidence for CSM interaction (Schlegel 1999). The typically faint X-ray luminosity is attributed to a low-density CSM surrounding the progenitor stars (Schlegel 2001). The presence of X-rays would likely indicate interaction with the CSM, which would also affect the UV luminosity. Thus, the lack of X-rays is consistent with the photospheric origin of the UV radiation discussed previously.

We calculated the upper limit to the mass-loss rate of the progenitor, assuming a shock velocity of $10,000 \mathrm{~km} \mathrm{~s}^{-1}$ and following the description by Immler et al. (2002). A $3 \sigma$ upper limit of $\dot{M} \lesssim 1 \times 10^{-5} M_{\odot} \mathrm{yr}^{-1}\left(v_{w} / 10 \mathrm{~km} \mathrm{~s}^{-1}\right)$, where $v_{w}$ corresponds to the wind velocity, is obtained for a median date of the XRT observations around $12 \pm 1$ days after the outburst. The upper limit to the mass-loss rate is consistent with those of core-collapse SN progenitors, which are in the range $10^{-6}$ to $10^{-4} M_{\odot} \mathrm{yr}^{-1}$ (Immler \& Lewin 2003) and $\sim 10$ times smaller than that of highly interacting SNe, such as SN 1979C (Immler et al. 2005b).

\section{SUMMARY}

SN 2005cs is the first SN IIP with a well-observed UV light curve and is an important addition to the SNe II observed in the UV by IUE and HST. The rapid drop in the UV shows the importance of quick response observations, as the emergent flux is dominated by the UV for only about a week after the explosion. The agreement of our CMFGEN models with the UVOT photometry and optical spectra (for a unique set of SN ejecta parameters), the identification of absorption lines in the UV (e.g., Mg II $2798 \AA$ ) and in the optical (e.g., H I Balmer lines with comparable Doppler-broadened widths), and the nondetection of X-rays all point to a photospheric origin of the UV emission and a lack of strong CSM interaction. The early UV photometry presented in this paper demonstrates the temporal effects of photospheric cooling and line blanketing, which can be reproduced by the non-LTE model atmosphere code CMFGEN. The effort to understand these effects will be continued by increasing the sample of UV light curves and spectra of SNe II with Swift in order to provide constraints for the spectroscopic modeling of multiple objects. These observations will also provide X-ray observations probing earlier times than have been studied in the past.

This work made use of the NASA IPAC Extragalactic Database. This work is supported at Pennsylvania State by NASA contract NAS5-00136. Supernova studies at Harvard University are supported by NSF grant AST 06-06772. R. P. K. acknowledges support from the Kavli Institute for Theoretical Physics through NSF grant PHY 99-07949. The F. L. Whipple Observatory is operated by the Smithsonian Astrophysical Observatory.
Barbon, R., Ciatti, F., \& Rosino, L. 1979, A\&A, 72, 2875 1982, A\&A, 116, 35

Baron, E. 2007, in The Multicoloured Landscape of Compact Objects and Their Explosive Progenitors: Theory versus Observations, ed. L. Burderi et al. (New York: AIP), in press (astro-ph/0611545)

Baron, E., Hauschildt, P. H., Branch, D., Kirshner, R. P., \& Filippenko, A. V. 1996, MNRAS, 279, 799

\section{REFERENCES}

Baron, E., et al. 1995, ApJ, 441, 170

2000, ApJ, 545, 444

Bianchi, L., et al. 2005, ApJ, 619, L71

Branch, D. 1987, ApJ, 20, L121

Brown, P. J., \& Immler, S. 2006, ATel, 761

Burrows, D., et al. 2005, Space Sci. Rev., 120, 165

Buta, R. J. 1982, PASP, 94, 578 
Calzetti, D., et al. 2005, ApJ, 633, 871

Cappellaro, E., Turatto, M., \& Fernley, J. 1995, IUE-ULDA Access Guide 6: Supernovae (ESA SP-1189; Noordwijk: ESA)

Cardelli, J. A., Clayton, G. C., \& Mathis, J. S. 1988, ApJ, 329, L33

Ciardullo, R., Feldmeier, J. J., Jacoby, G. H., Kuzio de Naray, R., Laychak, M. B., \& Durrell, P. R. 2002, ApJ, 577, 31

Crowther, P. A., Lennon, D. J., \& Walborn, N. R. 2006, A\&A, 446, 279

Dessart, L., \& Hillier, D. J. 2005, A\&A, 437, 667 2006, A\&A, 447, 691

Dewangan, G. C., Griffiths, R. E., Choudhury, M., Miyaji, T., \& Schurch, N. J. 2005, ApJ, 635, 198

Dickey, J. M., \& Lockman, F. J. 1990, ARA\&A, 28, 215

Feldmeier, J. J., Ciardullo, R., \& Jacoby, G. H. 1997, ApJ, 479, 231

Fransson, C. 1984, A\&A, 133, 264

Gehrels, N., et al. 2004, ApJ, 611, 1005

Georgiev, T. V., Getov, R. G., Zamanova, V. I., \& Ivanov, G. R. 1990, Soviet Astron. Lett., 16, 420

Hillier, D. J., \& Miller, D. L. 1998, ApJ, 496, 407

Immler, S., \& Brown, P. J. 2006, ATel, 776

Immler, S., Kong, A., \& Lewin, W. H. G. 2005a, IAU Circ. 8564

Immler, S., \& Lewin, W. 2003, in Supernovae and Gamma-Ray Bursters, ed. K. Weiler (Berlin: Springer), 91

Immler, S., Wilson, A. S., \& Terashima, Y. 2002, ApJ, 573, L27

Immler, S., et al. 2005b, ApJ, 632, 283 . 2006, ApJ, submitted

Jeffery, D. J., Ketchum, W., Branch, D., Baron, E., Elmhamdi, A., \& Danziger, I. J. 2006, ApJS, submitted (astro-ph/0607084)

Jeffery, D. J., et al. 1994, ApJ, 421, L27

Kirshner, R. P., Sonneborn, G., Crenshaw, D. M., \& Nassiopoulos, G. E. 1987, ApJ, 320, 602
Kloehr, W., Muendlein, R., Li, W., Yamaoka, H., \& Itagaki, K. 2005, IAU Circ. 8553

Leonard, D. C., et al. 2002a, AJ, 124, 2490 2002b, PASP, 114, 35

Li, W., Van Dyk, S. D., Filippenko, A. V., Cuillandre, J., Jha, S., Bloom, J. S., Riess, A. G., \& Livio, M. 2006, ApJ, 641, 1060

Lucy, L. B. 1987, A\&A, 182, L31

Maund, J. R., Smartt, S. J., \& Danziger, I. J. 2005, MNRAS, 364, L33

Mazzali, P. A., \& Lucy, L. B. 1993, A\&A, 279, 447

Modjaz, M., Kirshner, R., Challis, P., \& Hao, H. 2005, IAU Circ. 8491

Nota, A., Jedrezejewski, R., Voit, M., \& Hack, W. 1996, FOC Instrument Handbook Version 7.0 (Baltimore: STScI)

Panagia, N., et al. 1980, MNRAS, 192, 861

Pastorello, A., et al. 2006, MNRAS, 370, 1752

Pooley, D., \& Lewin, W. H. G. 2004, IAU Circ. 8390

Pooley, D., et al. 2002, ApJ, 572, 932

Pun, C. S. J., et al. 1995, ApJS, 99, 223

Richmond, M. W., Treffers, R. R., Filippenko, A. V., Paik, Y., Leibundgut, B., Schulman, E., \& Cox, C. V. 1994, AJ, 107, 1022

Roming, P. W. A., et al. 2005, Space Sci. Rev., 120, 95

Sandage, A., \& Tammann, G. A. 1974, ApJ, 194, 559

Schlegel, D. J., Finkbeiner, D. P., \& Davis, M. 1998, ApJ, 500, 525

Schlegel, E. M. 1999, ApJ, 527, L85 2001, ApJ, 556, L25

Takáts, K., \& Vinkó, J. 2006, MNRAS, 372, 1735

Tonry, J. L., Dressler, A., Blakeslee, J. P., Ajhar, E. A., Fletcher, A. B., Luppino, G. A., Metzger, M. R., \& Moore, C. B. 2001, ApJ, 546, 681

Tsvetkov, D. Y., Volnova, A. A., Shulga, A. P., Korotkiy, S. A., Elmhandi, A., Danziger, I. J., \& Ereshko, M. V. 2006, A\&A, 460, 769 